\title{
Antibiogram of Biofilm Producing and Non-Producing Community Acquired-Methicillin Resistant Staphylococcus aureus Isolated from Potential Risk Population of Dharan, Nepal
}

Jenish Shakya

Central Campus of Technology,TU https://orcid.org/0000-0002-9397-4511

Bijay Kumar Shrestha

Central Campus of Technology, TU

\section{Prince Subba}

Central Campus of Technology

Hemanta Khanal ( $\sim$ khanal.hemanta@gmail.com )

https://orcid.org/0000-0002-8078-7039

\section{Research article}

Keywords: CA-MRSA, biofilm, antibiotic susceptibility test, potentially risk population

Posted Date: September 8th, 2019

DOI: https://doi.org/10.21203/rs.2.14153/v1

License: (c) (7) This work is licensed under a Creative Commons Attribution 4.0 International License. Read Full License 


\section{Abstract}

Background Staphylococcus aureus is one of the common cause of hospital acquired infection and community acquired infections. Nowadays these organisms became resistant towards variety of drugs. MRSA is the emerging antibiotic resistant bacteria that are resistant to methicillin antibiotic and known to be the infectious pathogen causing severe infection and a cause of fatal mortality.Materials and methodology Altogether 200 nasal swabs and 200 hand swabs were obtained from participants and transported to microbiology lab in cold chain. The samples were swabbed in mannitol salt agar (MSA) containing oxacillin powder of $6 \mathrm{mg} / \mathrm{L}$ and incubated at $37^{\circ} \mathrm{C}$ for $24 \mathrm{hrs}$. Staphylococcus aureus colonies were identified based on growth characteristics on MSA plates (golden yellow colonies), Gram stain and positive results for coagulase tube test and catalase test. The pure isolated MRSA were subjected to antibiotic susceptibility tests and biofilm formation assays.

Results From our study the overall prevalence of CA-MRSA was $61.5 \%$. Higher frequency of multi-drug resistant MRSA was isolated. The biofilm producing CA-MRSA were $51.2 \%$ which showed high drug resistance and rest (48.7\%) were non-biofilm producers. There was significant association in biofilm production with multi-drug resistance $(p<0.05)$. Ciprofloxacin was most sensitive drug against the isolates which was statistically significant $(p<0.05)$. The resistant pattern of biofilm producers reported high ability of multi-drug resistance compared to nonbiofilm producers $(p<0.05)$. Microtitre plate method was found to be gold standard over tube and congo red agar method for screening biofilm formation. Surprisingly the emergence of VISA and VRSA strains were significantly reported from our study. The prevalence of VISA and VRSA among CA-MRSA was found to be $49.5 \%$ and $40.6 \%$ respectively among the isolates which indicates the failure of Vancomycin drug in clinical therapy.

Conclusions The prevalence of CA-MRSA was found more in barbers followed by beauticians and municipal waste workers in comparison to healthy controls. This study reported the higher carriage of CA-MRSA in potential risk population along with emergence of VISA and VRSA strains. Improvement in personal hygiene and formulation of appropriate health policy helps to prevent CA-MRSA infection. This study concludes that CA-MRSA is still emerging with multi-drug resistance.

\section{Background}

Methicillin-resistant Staphylococcus aureus (MRSA) strains or multidrug-resistant S. aureus is pathogenic bacteria responsible for rapid progressive fatal diseases including life-threatening pneumonia, necrotizing fasciitis, endocarditis, osteomyelitis, severe sepsis, and toxic shock syndrome [1]. MRSA bacteria are well studied organism of medical significance because of its wide resistance to many antibiotics of group's aminoglycosides, macrolides, fluoroquinolones, chloramphenicol, and tetracycline [2]. CA-MRSA has been known to be the emerging superbug as a causative agent of skin and soft-tissue infections [3]. These strains became resistant to penicillin by producing a plasmid-encoded penicillinase, called $\beta$-lactamase that can break down the $\beta$-lactam ring of penicillin, making it ineffective [4]. Smyth et al. 2005 reported that MSA containing $6 \mathrm{mg} /$ liter cefoxitin allowed the isolation of all MRSA [5]. Biofilm producing MRSA infections are life threatening infection because of its multi-drug resistance and resistance of biofilm on the action of antibiotics biofilm and are of significance in medical science. Vancomycin has been a drug of choice for the treatment of methicillin-resistant Staphylococcus aureus (MRSA) infection. The limitation of other antibiotics induces the emergence of VRSA is making the treatment of MRSA difficult [6]. The emergence of VISA strains and VRSA strains has been reported where the higher MIC of Vancomycin resistant of MRSA has been known to be associated with drug resistance to many other antibiotics [7]. Hence, the main aim of 
this study was to find the prevalence and antibiogram of CA-MRSA colonizing hand and nostril of potentially risk population of eastern Nepal.

\section{Methods}

\section{Research Design}

The study was conducted from June to November 2018 after receiving Ethical approval (Reg. No-297/2018) from Nepal Health Research Council (NHRC) on 20 June 2018. This study was a laboratory based cross-sectional study. Potential risk population's municipal waste workers, barbers, beauticians and physically healthy (controls) were participated in the research. All the sampling populations were selected from Dharan Sub-Metropolitan city by simple random sampling. All the work concerning this research was carried out in microbiology laboratory of Central Campus of Technology, Dharan.

\section{Laboratory methods}

Samples were collected from potential risk population of Dharan. Samples taken for this study were skin and nasal swabs. Nasal and skin swabs were taken by using sterile swabs in $10 \mathrm{~mL}$ nutrient broth. Nasal swab and skin swab samples were collected with sterile disposable cotton swabs (HiMedia, India) and was stored in a sterile vial and transported in cold chain to microbiology Lab. The MRSA isolates were isolated and identified according to Smyth et al. 2005 [5]. The samples were swabbed in MSA containing oxacillin powder of $6 \mathrm{mg} / \mathrm{L}$ and incubated at $37^{\circ} \mathrm{C}$ for 24 hours. MRSA were identified based on evidence from growth on MSA media containing oxacillin powder, Gram's staining, catalase test, and coagulase test. Further the isolates were confirmed to bae MRSA by cefoxitin $(30 \mu \mathrm{g})$ disk diffusion method according to Clinical standard Institute guidelines 2012 [8].

\section{Antibiotic susceptibility testing}

All MRSA isolated from clinical samples were subjected to in-vitro antibiotic susceptibility test by Kirby-Bauer disc diffusion techniques using Mueller Hinton agar (MHA) (HiMedia Mumbai, India) containing $4 \% \mathrm{NaCl}$ as recommended by CLSI 2012 [8]. Fresh colonies were selected and transferred into NB to obtain turbidity equivalent to $0.5 \mathrm{McF}$ arland barium sulfate standards $\left(1.5 \times 10^{8} \mathrm{CFU} / \mathrm{ml}\right)$. MHA plates were inoculated with sterile cotton swabs then antibiotics were placed with sterile forceps and allowed to stand at room temperature for 15 minutes for pre-diffusion then incubated at $37^{\circ} \mathrm{C}$ for $16-18$ hours. The zone of inhibition was interpreted as susceptible, intermediate and resistant according to CLSI "Diffusion Supplemental Table" (2012). In this study the antibiotics used Amoxicillin (AMX, 10mcg), Ampicillin (AMP, 10mcg), Cefoxitin (CX, 30mcg), Cefotaxime (CTX, 30mcg), Chloramphenicol (C, 30mcg), Ciprofloxacin (CIP, 5mcg), Co-Trimoxazole (COT, 25mcg), Erythromycin (E, 15mcg), Gentamicin (GEN, 10mcg), Norfloxacin (NX,10mcg), Ofloxacin (OF, 5mcg), Teicoplanin (TEl, 30mcg), Trimethoprim (TR, 5mcg), Tetracycline (TE, 30mcg) (Himedia, Mumbai, India).

\section{Biofilm formation test}

\section{Microtitre plate method}

The quantification of biofilm by microtitre plate was performed according to Christensen et al. 1985 [9]. In this method, $5 \mathrm{ml}$ of overnight culture of MRSA was prepared. Then 100 microliter of diluted culture was inoculated in a microtitre well containing TSB with glucose. The plate was incubated at $37^{\circ} \mathrm{C}$ for 24 hours for biofilm production. The unbound cell was discarded and washes several times by PBS (pH-7.2). $125 \mu$ of $0.1 \%$ crystal-violet solution 
was added and left for 10-15 minutes incubation. The plate was washed and left inverted for dry. The quantitative determination was performed by solubilizing the biofilm by adding $125 \mu$ of $30 \%$ acetic acid to each well and incubated the plate for 10-15 minutes at room temperature and transfer to another microtitre plates and reading the absorbance at $570 \mathrm{~nm}$ by ELISA plate reader. The interpretation of biofilm production was done according to the criteria of Stepanovic et al. (2007) [10]. Interpretation is made on optical density (OD) of test wells. The optical density $\left(O D_{s}\right)$ of each strain was obtained by the arithmetic mean of the absorbance of three wells and this value was compared with the mean absorbance of negative controls $\left(O D_{n c}\right)$. The following classification was used for the determination of biofilm formation: no biofilm production $\left(O D_{s} \leq O D_{n c}\right)$, weak biofilm production $\left(O D_{n c}<O D_{s} \leq 2 . O D_{n c}\right)$, moderate biofilm production $\left(2.0 D_{n c}<O D_{s} \leq 4 . O D_{n c}\right)$ and strong biofilm production $\left(4.0 D_{n c}<O D_{s}\right)[10]$.

\section{Tube method}

A qualitative assessment of biofilm formation was done as described by Christensen et al. 1985 [9]. The TSB glucose $(10 \mathrm{~mL})$ was inoculated with a loop full of MRSA from overnight culture plates and incubated for 24 hours at $37^{\circ} \mathrm{C}$. The tubes were decanted and washed with PBS $(\mathrm{pH}-7.2)$ and dried. Then the tubes were stained by $0.1 \%$ crystal violet. Stain was removed by deionized water. Tubes were then dried in inverted position for biofilm formation. Biofilm formation was considered positive when a visible film lined the wall and bottom of the tube. Ring formation at the liquid interface was not indicative of biofilm formation. Experiments was performed in triplicate and repeated for three times.

\section{Congo red agar method (CRA)}

The congo red agar method was performed according to Freeman et al. 1989 [11]. The MRSA culture was streaked on surface of congo red agar and incubated at $37^{\circ} \mathrm{C}$ for $24-48$ hours. Black coloured colonies with dry crystalline consistency interpreted as positive biofilm producing strains. Red coloured colonies - interpreted as negative for biofilm production.

\section{Determination of Minimum Inhibitory Concentration}

The minimum inhibitory concentration of vancomycin (HiMedia, Mumbai, India) to MRSA isolates were screened by Microdilution method as suggested by CLSI 2012 [8]. The vancomycin powder was accurately weighed and stock solution of $128 \mu \mathrm{g} / \mathrm{ml}$ was prepared. The known volume of $0.5 \mathrm{McF}$.rland suspension of bacterial culture was added in each well containing the trypticase soya broth (TSB). From the stock solution the different concentrations of drug ranging from $64 \mu \mathrm{g} / \mathrm{ml}$ to $0.125 \mu \mathrm{g} / \mathrm{ml}$ was made in in round bottom microtitre plates by serial dilution. The wells for positive and negative controls were even maintained in the plates. The microtitre plates were incubated at $37^{\circ} \mathrm{C}$ for 24 hours. The well with concentration of drug in which the growth of bacteria was inhibited was known to be the MIC.

\section{Data analysis}

The information collected from schedule was documented and tabulated. The data were statistically analyzed at $5 \%$ level of significance by SPSS version 16. A p-value of less than or equal to 0.05 was considered to be statistically significant $(\mathrm{p} \leq 0.05)$.

\section{Results}


Gender-wise distribution of MRSA in sample population

Out of 400 samples taken, 123 were MRSA positive isolates out of which 67 (54.4\%) were from males and 56 (45.5\%) were from females.

Table 1: Gender-wise distribution of MRSA in sample population

\begin{tabular}{ll}
\hline Gender & Presence\% (n) \\
\hline Male (105) & $67(63.80 \%)$ \\
Female (95) & $56(58.94 \%)$ \\
\hline Total 200 & 123 \\
\hline
\end{tabular}

Gender-wise distribution of MRSA positive in sample population

Out of 400 samples taken, 123 were MRSA positive isolates among which 34 MRSA were isolated from barber's populations. Similarly, 32 MRSA were isolated from beautician's populations. In municipal waste worker's population, 19 MRSA isolates were obtained from males whereas 10 were isolated from female's population whereas in healthy population, 14 MRSA isolates were obtained from males and 14 were from female's population.

\section{Comparative study of MRSA isolated from total samples}

In this study, all samples were analyzed for MRSA. Higher number of MRSA was isolated from hand (36.5\%). The prevalence of MRSA isolates from nasal was $25 \%$ and from hand was $36.5 \%$.

Table 2: Comparative study of MRSA isolated from total samples

\begin{tabular}{cccc}
\hline Samples & Total sample & MRSA & p-value \\
\hline Hand & 200 & $73(36.5 \%)$ & 0.013 \\
Nasal & 200 & $50(25 \%)$ & \\
\hline Total & 400 & 123 & \\
\hline
\end{tabular}

\section{Comparative study of MRSA isolated from different samples}

The MRSA isolated from hand were 73 and from nasal were 50. In 24 individuals the MRSA was isolated from both the hand and nasal. The higher frequency of MRSA was isolated from hand than in nasal. Maximum MRSA isolates were obtained from skin surface of barber's hand.

Table 3: Comparative study of MRSA isolated from different samples

\begin{tabular}{lcccc}
\hline Samplepopulation & \multicolumn{3}{c}{ Source } & \\
\cline { 2 - 4 } & Hand (Only) & Nasal (Only) & Both & p-value \\
\hline Barber & 23 & 11 & 8 & 0.011 \\
Beauticians & 19 & 13 & 4 & 0.086 \\
Municipal waste workers & 16 & 13 & 4 & 0.509 \\
Healthy & 15 & 13 & 8 & 1.00 \\
\hline Total & 73 & 50 & 24 & \\
\hline
\end{tabular}




\section{MRSA isolated from different age groups of male and female}

Among 123 MRSA isolated, MRSA from male were 67 (54.4\%) and MRSA were from female 56 (45.5\%). In male the highest number of MRSA, 27 (40.2\%) was from the age group of 20-29 years followed by 25 (37.3\%) from 30-39 years of age. In female the highest number of MRSA, 18 (32.1\%) was isolated from age group of 20-29 years and 30-39 years of age.

Table 4: MRSA isolated from different age groups of male and female

\begin{tabular}{|c|c|c|c|c|c|c|c|c|}
\hline \multirow{2}{*}{$\begin{array}{c}\text { Age } \\
\text { Groups }\end{array}$} & \multicolumn{8}{|c|}{ Prevalence of MRSA } \\
\hline & $\begin{array}{c}\text { Barbers } \\
\text { Males }\end{array}$ & $\begin{array}{l}\text { Barbers } \\
\text { Females }\end{array}$ & $\begin{array}{l}\text { Beauticians } \\
\text { Males }\end{array}$ & $\begin{array}{c}\text { Beauticians } \\
\text { Females }\end{array}$ & $\begin{array}{c}\text { Municipal } \\
\text { Waste } \\
\text { Workers } \\
\text { Males }\end{array}$ & $\begin{array}{c}\text { Municipal } \\
\text { Waste } \\
\text { Workers } \\
\text { Females }\end{array}$ & $\begin{array}{l}\text { Healthy } \\
\text { Males }\end{array}$ & $\begin{array}{l}\text { Healthy } \\
\text { Females }\end{array}$ \\
\hline $\begin{array}{c}\text { Below } \\
20\end{array}$ & $10.4 \%$ & - & $\begin{array}{ll}- \\
-\end{array}$ & $5.3 \%$ & 0.0 & 0.0 & $2.9 \%$ & $1.7 \%$ \\
\hline $20-29$ & $17.9 \%$ & - & - & $17.8 \%$ & $8.9 \%$ & $1.7 \%$ & $13.4 \%$ & $12.5 \%$ \\
\hline $30-39$ & $16.4 \%$ & - & - & $21.4 \%$ & $17.9 \%$ & $10.7 \%$ & $2.9 \%$ & 0.0 \\
\hline $40-49$ & $4.4 \%$ & - & - & $10.7 \%$ & $1.4 \%$ & $5.3 \%$ & 0.0 & $5.3 \%$ \\
\hline $\begin{array}{c}\text { Above } \\
50\end{array}$ & $1.4 \%$ & - & - & $1.7 \%$ & 0.0 & 0.0 & $1.4 \%$ & $5.3 \%$ \\
\hline
\end{tabular}

\section{Comparative study of biofilm formation by MRSA in potential risk population}

The biofilm formation assay showed that maximum isolates were biofilm producer. The biofilm producing MRSA was found maximum in beautician $(56.2 \%)$, followed by barber (52.9\%). Similarly, in municipal waste workers and healthy population biofilm producing MRSA were $48.2 \%$ and $46.4 \%$ respectively.

Table 5: Comparative study of biofilm formation by MRSA in potential risk population

\begin{tabular}{llllll}
\hline Biofilm & Barber & Beautician & Municipal waste workers & Healthy & p-value \\
\hline Strong & $7(20.5 \%)$ & $5(15.6 \%)$ & $2(6.8 \%)$ & $5(17.8 \%)$ & 0.032 \\
Moderate & $11(32.3 \%)$ & $13(40.6 \%)$ & $12(41.3 \%)$ & $8(28.5 \%)$ & 0.033 \\
Weak & $16(47 \%)$ & $14(43.7 \%)$ & $15(51.7 \%)$ & $15(53.5 \%)$ & 0.045 \\
\hline Total & 34 & 32 & \multicolumn{2}{c}{29} & 28 \\
\hline
\end{tabular}

\section{Biofilm formation assay}

The biofilm forming ability of isolated MRSA was performed by three methods: microtitre plate method, tube method and congo red agar method.

Table 6: Biofilm formation by MRSA by three methods 


\begin{tabular}{lcccc}
\hline $\begin{array}{l}\text { Biofilm } \\
\text { formation }\end{array}$ & $\begin{array}{c}\text { Microtitre plate } \\
\text { method }\end{array}$ & $\begin{array}{c}\text { Tube } \\
\text { method }\end{array}$ & $\begin{array}{c}\text { Congo red agar } \\
\text { method }\end{array}$ & p-value \\
\hline High & $19(15.4 \%)$ & $14(11.3 \%)$ & $11(8.9 \%)$ & \\
Moderate & $44(35.7 \%)$ & $37(30 \%)$ & $31(25.2 \%)$ & 0.000 \\
Weak/None & $60(48.7 \%)$ & $72(58.5 \%)$ & $81(65.8 \%)$ & \\
\hline Total Isolates & 123 & 123 & 123 & \\
\hline
\end{tabular}

\section{Sensitivity and specificity of biofilm screening methods}

The microtitre plate method was found to be most efficient standard method for studying biofilm formation as compared to tube method and congo red agar method. The parameters like sensitivity, specificity, negative predictive value, positive predictive value and accuracy were calculated. True positives were biofilm producers by microtitre, tube and congo red agar method. False positive were biofilm producers by tube method (TM) and congo red agar (CRA) method and not by microtitre method. False negative were the isolates which were non-biofilm producers by microtitre plate and CRA but were biofilm producer by microtitre method. True negatives are those which were non biofilm producers by all three methods [12].

Table 7: Sensitivity and specificity of biofilm screening methods

\begin{tabular}{lccccc}
\hline Biofilm screening method & $\begin{array}{c}\text { Sensitivity } \\
(\%)\end{array}$ & $\begin{array}{c}\text { Specificity } \\
(\%)\end{array}$ & $\begin{array}{c}\text { PPV } \\
(\%)\end{array}$ & $\begin{array}{c}\text { NPV } \\
(\%)\end{array}$ & Accuracy (\%) \\
\hline Tube method & 75 & 74.6 & 58.8 & 86.1 & 64.2 \\
Congo red agar method & 54.8 & 85.2 & 79 & 65 & 21.4 \\
\hline
\end{tabular}

Antibiotic susceptibility pattern of MRSA isolates

The provided table shows the resistance and sensitivity pattern of isolated MRSA strains towards different antibiotics. It was found that MRSA was most sensitive towards ciprofloxacin (93.4\%), chloramphenicol (80.4\%) and co-trimoxazole $(74.7 \%)$ which was statistically significant $(P<0.05)$. The isolated MRSA were resistant to ampicillin (100\%), amoxicillin (100\%) and trimethoprim (23.5\%).

Table 8: Antibiotic susceptibility pattern of MRSA isolates

\begin{tabular}{|c|c|c|c|c|c|}
\hline \multirow[t]{2}{*}{ Antibiotics } & \multicolumn{2}{|c|}{ Resistant } & \multicolumn{2}{|c|}{ Sensitive } & \multirow[t]{2}{*}{ p-value } \\
\hline & MRSA & $\%$ & MRSA & $\%$ & \\
\hline Ampicillin & 123 & 100 & 0 & 0 & 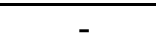 \\
\hline Amoxicillin & 123 & 100 & 0 & 0 & - \\
\hline Cefotaxime & 65 & 52.8 & 58 & 47.1 & 0.00 \\
\hline Chloramphenicol & 25 & 20.3 & 98 & 80.4 & 0.00 \\
\hline Ciprofloxacin & 8 & 6.5 & 115 & 93.4 & 0.00 \\
\hline Co-Trimoxazole & 31 & 25.2 & 92 & 74.7 & 0.00 \\
\hline Erythromycin & 90 & 73.1 & 33 & 26.8 & 0.01 \\
\hline Gentamicin & 42 & 34.1 & 81 & 65.8 & 0.04 \\
\hline Norfloxacin & 76 & 61.7 & 47 & 38.2 & 0.00 \\
\hline Ofloxacin & 38 & 30.8 & 85 & 69.1 & 0.00 \\
\hline Teicoplanin & 55 & 44.7 & 68 & 55.2 & 0.82 \\
\hline Tetracycline & 59 & 47.9 & 64 & 52 & 0.62 \\
\hline Trimethoprim & 94 & 76.4 & 29 & 23.5 & 0.00 \\
\hline
\end{tabular}




\section{Resistance pattern of biofilm producing MRSA}

The biofilm producing MRSA showed resistance to cefotaxime, chloramphenicol, teicoplanin, co-trimoxazole, erythromycin, norfloxacin, trimethoprim. The non-biofilm producing MRSA showed resistance to tetracycline, ofloxacin, gentamicin, and ciprofloxacin. Ampicillin and amoxicillin were resisted by both biofilm producers and non-biofilm producers.

Table 9: Resistance pattern of biofilm producing MRSA

\begin{tabular}{lcc}
\hline Antibiotics & \% of biofilm producing resistant & \% of non-biofilm producing resistant \\
\hline Ampicillin & $100 \%$ & $100 \%$ \\
Amoxicillin & $100 \%$ & $100 \%$ \\
Cefotaxime & $56.6 \%$ & $38 \%$ \\
Chloramphenicol & $20.6 \%$ & $15 \%$ \\
Ciprofloxacin & $1.5 \%$ & $10 \%$ \\
Co-trimoxazole & $25.3 \%$ & $25 \%$ \\
Erythromycin & $73 \%$ & $65 \%$ \\
Gentamicin & $33.3 \%$ & $38.3 \%$ \\
Norfloxacin & $66.6 \%$ & $60.3 \%$ \\
Ofloxacin & $25.3 \%$ & $36.6 \%$ \\
Teicoplanin & $48.3 \%$ & $34.9 \%$ \\
Tetracycline & $30.1 \%$ & $55 \%$ \\
Trimethoprim & $78.3 \%$ & $77.7 \%$ \\
\hline
\end{tabular}

\section{Multidrug resistant (MDR) CA-MRSA}

Multidrug resistant community-acquired methicillin resistant $S$. aureus (CA-MRSA) were identified by their antibiotic resistivity pattern on, three or more than three commonly prescribed antibiotics of different classes. The prevalence of MDR CA-MRSA was $91 \%$.

Table 10: Multidrug resistant (MDR) CA-MRSA

\begin{tabular}{lc}
\hline Isolates & CA-MRSA \\
\hline Total isolates & 123 \\
Multi-drug resistant & $112(91 \%)$ \\
\hline
\end{tabular}

\section{Vancomycin sensitivity of total MRSA isolates}

The MIC of vancomycin to the MRSA isolates was screened by microbroth dilution assay performed in microtitre wells. In our study, out of 123 MRSA samples the VSSA isolates were $9.7 \%$, VISA isolates were $49.5 \%$ and VRSA isolates were $40.6 \%$. In our study the high prevalence of VISA and VRSA isolates were screened.

MIC of vancomycin to MRSA isolates from different sample population 
In our study the VSSA, VISA and VRSA strains of MRSA were screened from all sample populations under study. The highest prevalence of VISA strain was found in healthy population (15.4\%). The highest prevalence of VRSA was found in barbers (15.4\%).

Table 11: MIC of vancomycin to MRSA isolates from different sample population

\begin{tabular}{|c|c|c|c|c|}
\hline \multirow[t]{2}{*}{ Sample population } & \multicolumn{3}{|c|}{ MIC Vancomycin } & \multirow[t]{2}{*}{ Total } \\
\hline & VSSA( $\leq 2 \mu \mathrm{g} / \mathrm{ml})$ & VISA(4-8 $\mu \mathrm{g} / \mathrm{ml})$ & VRSA $(\geq 16 \mu \mathrm{g} / \mathrm{ml})$ & \\
\hline MWW & $2(1.6 \%)$ & $18(14.6 \%)$ & $9(7.3 \%)$ & $29(23.5 \%)$ \\
\hline Barbers & $3(2.4 \%)$ & $12(9.7 \%)$ & $19(15.4 \%)$ & $34(27.6 \%)$ \\
\hline Beauticians & $4(3.2 \%)$ & $12(9.7 \%)$ & $16(13 \%)$ & $32(26 \%)$ \\
\hline Healthy & $3(2.4 \%)$ & $19(15.4 \%)$ & $7(5.6 \%)$ & $28(22.7 \%)$ \\
\hline Total & 12 & 61 & 50 & 123 \\
\hline
\end{tabular}

MIC of vancomycin to MRSA isolates from different gender population

The highest prevalence of VISA and VRSA were found to be in male population than in female population. However, the sample populations of female were less than that of male in our study.

Table 12: MIC of vancomycin to MRSA isolates from different gender population

\begin{tabular}{lcccc}
\hline MIC vancomycin & Male & Female & Total & p-value \\
\hline VSSA ( $\mathbf{2} \mathbf{\mu} \mathbf{g} / \mathrm{ml})$ & $5(4 \%)$ & $7(5.6 \%)$ & 12 & 0.348 \\
VISA (4-8 $\mathbf{\mu g} / \mathbf{m l})$ & $33(26.8 \%)$ & $28(22.7 \%)$ & 61 & 0.113 \\
VRSA ( $\mathbf{1 6} \mathbf{\mu} \mathbf{g} / \mathbf{m l})$ & $29(23.5 \%)$ & $21(17 \%)$ & 50 & 0.515 \\
\hline Total & 67 & 56 & 123 & \\
\hline
\end{tabular}

\section{MIC of vancomycin to MRSA isolates from hand and nasal sample}

In this study, the highest prevalence of VISA was found to be in hand (31.7\%) than in nasal (17.8\%). Similarly, the highest prevalence of VRSA was found to be in hand (21.9\%) than in nasal (18.6\%). However, VSSA isolates were found more in hand (5.6\%) sample than in nasal (4\%) sample.

Table 13: MIC of vancomycin to MRSA isolates from hand and nasal sample

\begin{tabular}{lcccc}
\hline MIC vancomycin & Hand & Nasal & Total & p-value \\
\hline VSSA ( $\mathbf{2} \mathbf{\mu} \mathbf{g} / \mathrm{ml})$ & $7(5.6 \%)$ & $5(4 \%)$ & 12 & 0.940 \\
VISA $(\mathbf{4 - 8} \mathbf{\mu g} / \mathrm{ml})$ & $39(31.7 \%)$ & $22(17.8 \%)$ & 61 & 0.304 \\
VRSA ( $\mathbf{1 1 6} \mathbf{\mu g} / \mathbf{m l})$ & $27(21.9 \%)$ & $23(18.6 \%)$ & 50 & 0.317 \\
\hline Total & 73 & 50 & 123 & \\
\hline
\end{tabular}

MIC of vancomycin to MRSA isolates from different age groups of sample population

Among 123 MRSA isolates, the highest VISA isolates were screened from the age group 20-29 years (21.1\%). Even the highest percentage of VRSA was screened from the age group of $20-29$ years (14.6\%). 
Table 14: MIC of vancomycin to MRSA isolates from different age groups of sample population

\begin{tabular}{lcccc}
\hline Age group & $\begin{array}{c}\text { VSSA } \\
(\leq 2 \mu \mathrm{g} / \mathrm{ml})\end{array}$ & $\left.\begin{array}{c}\text { VISA } \\
\text { (4-8 } \mathbf{\mu g} / \mathrm{ml})\end{array}\right)$ & $\begin{array}{c}\text { VRSA } \\
(\mathbf{1 6 \mu g} / \mathrm{ml})\end{array}$ & Total \\
\hline Below 20 Years & 0 & $10(8.1 \%)$ & $10(8.1 \%)$ & $20(16.2 \%)$ \\
20-29 Years & $3(2.4 \%)$ & $26(21.1 \%)$ & $18(14.6 \%)$ & $47(38.2 \%)$ \\
30-39 Years & $2(1.6 \%)$ & $15(12.1 \%)$ & $15(12.1 \%)$ & $32(26 \%)$ \\
40-49 Years & $2(1.6 \%)$ & $8(6.5 \%)$ & $6(4.8 \%)$ & $16(13 \%)$ \\
50 and Above & $5(4 \%)$ & $2(1.6 \%)$ & $1(0.8 \%)$ & $8(6.5 \%)$ \\
\hline Total & 12 & 61 & 50 & 123 \\
\hline
\end{tabular}

\section{Discussion}

Staphylococcus aureus is one of the common pathogens isolated in most microbiological laboratories [13]. It is responsible for a wide range of infections including superficial skin infections, food poisoning, osteomyelitis and septicemia [14].

Methicillin-resistant strains of staphylococci emerged by late 1970s and now have stood as prevalent as nosocomial pathogens [15]. The emergence of vancomycin resistant $S$. aureus has raised the concern that none of the antimicrobial drugs will be left to treat patients infected by these strains of staphylococci [16].

Our study was carried out at microbiology laboratory of Central Campus of Technology, Hattisar, Dharan, Submetropolitan city. During the study period, two types of samples were collected from single individual under study and processed for the isolation and identification of CA-MRSA. The sample included nasal swab and hand swab from each person. In our study 123 community acquired methicillin resistant Staphylococcus aureus (CA-MRSA) was isolated from 400 samples in which 200 were nasal swab sample and 200 were hand swab sample. In this study the total identified MRSA was 123 (30.7\%) isolated from 400 samples. In our study the prevalence of MRSA in 200 sample population was found to be $61.5 \%$. The prevalence of MRSA in Nepal ranges from 39\% to 69\% [17, $18,19,20,21]$ which showed similar result to our study.

In our study higher frequency of MRSA was isolated from males than in females, 67 (63.80\%) from males and 56 (58.94\%) from females. Prevalence of MRSA in male was higher than in female according to previous study done by Khanal and Jha. 2010 [20]. Hence, prevalence of MRSA was seen more in males as compared with females. Similar findings were obtained even in our study although the prevalence of MRSA in two gender was not found statistically significant, $p>0.05$.

In male, the prevalence of MRSA was highest with 27 (40.2\%) in age group 20-29 years followed by 25 (37.3\%) from 30-39 years of age. In female, the prevalence of MRSA was highest in age group of 20-29 years of age with 18 (32.1\%) positive samples. However, the prevalence of MRSA among different age group was not statistically significant, $p>0.05$. In our study the highest percentage of MRSA was isolated from hand $73(36.5 \%)$ and in nasal it was found to be 50 (25\%). Lower frequency of nasal carriage of MRSA was found in the studies performed by Rai et al. 1990 that reported $7.5 \%$ nasal carriage [16]. The prevalence of CA-MRSA in hand and nasal was statistically significant, $p<0.05$.

From 50 barber's population the total samples obtained were 100 (50 hand and 50-nasal). Out of total barbers samples 34 MRSA were isolated; 23 (46\%) from hand and 11 (22\%) from nasal. In beauticians, 32 MRSA were isolated; 19 (38\%) from hand and 13 (26\%) from nasal. In municipal waste workers the isolated MRSA were 29 out of which $16(32 \%)$ were from hand swab and 13 (26\%) were from nasal swab. In healthy population isolated MRSA 
were 28 out of which 14 (28\%) were from hand swab and 14 (28\%) were from nasal swab. The frequency of isolated MRSA was reported more from the barbers followed by beauticians and municipal waste workers. This study reported the carriage of CA-MRSA higher in potential risk population. The prevalence of MRSA in hand and nasal of Barber's population was statistically significant $(p<0.05)$.

Presence of MRSA in waste workers may be due to the direct contact with contaminated clinical wastes. There was statistical significant relation between presence of MRSA and workers exposure to clinical wastes $(p-v a l u e<0.05)$. Municipal waste workers were under regular exposure to clinical and domestic wastes which could be the reason behind higher colonization of MRSA in municipal waste workers. Prevalence of MRSA with the previous history of skin infection in the population was found to be statistically significant ( $p$-value<0.05).

Risk factors of CA-MRSA in skin infections include exposure to clinical wastes, occupations, skin to skin contact, irrational use of antibiotics, old age, etc. For the treatment of MRSA infection the most often used drug include vancomycin but the emergence of vancomycin resistant Staphylococcus aureus (VRSA) has become a great issue as it leads to failure of clinical therapy in treating infections.

CDC estimated that about $12 \%$ of MRSA infections are now community associated but this percentage can vary by community and patient population due to the recent antibiotic use, sharing contaminating items, having active skin diseases or injuries, poor hygiene and living in crowded settings. The transmission of MRSA is largely from people with active MRSA skin infections and spread always by four routes of transmission of MRSA. They are direct contact with infected or colonized individuals, indirect contact through contaminated hands of health care workers, contaminated air and contaminated environmental surfaces or equipment.

In our study, the antibiotic susceptibility test of CA-MRSA reported that the most effective antibiotic was ciprofloxacin, chloramphenicol and co-trimaxole. Out of 123 CA-MRSA positive samples, ciprofloxacin was $93.4 \%$ sensitive, chloramphenicol was $80.4 \%$ sensitive and co-trimoxazole was $74.7 \%$ sensitive. The sensitivity of the MRSA against those drugs was statistically significant $(\mathrm{p}<0.05)$. Ampicillin and amoxicillin were $100 \%$ resistant. Other antibiotics such as trimethoprim (23.5\%), erythromycin (26.8\%), norfloxacin (38.2\%) and cefotaxime (47.1\%) showed less than $50 \%$ sensitivity whereas; tetracycline (52\%), teicoplanin (55.2\%), gentamicin (65.8\%), and ofloxacin (69.1\%) showed more than 50\% sensitivity. In a study conducted by Wylie et al. 2005 erythromycin susceptibility was $40 \%$ to CA-MRSA [22]. Erythromycin susceptibility of CA-MRSA strains was $51.7 \%$ as done by Mandelia et al. 2012 [23]. Around 73.1\% of CA-MRSA isolates were resistant to erythromycin in our study. Our result supports the previous studies conducted in Nepal which reported increasing resistance of bacteria towards erythromycin and co-trimoxazole due to excessive use of their drugs to treat infections [21]. Even in our study the isolated superbug showed reduced sensitivity to erythromycin. The susceptibility of ciprofloxacin was $88 \%$ observed in study conducted by Khatri et al. 2017 [24]. In our study the susceptibility to ciprofloxacin was $93.4 \%$ which was found high in comparison to other studies. Isolates MRSA were most sensitive to ciprofloxacin drug which was statistically significant with $p<0.05$. In study conducted by Mandelia et al. (2012) the susceptibility to ciprofloxacin was only $18.3 \%$ [23]. Chloramphenicol, co-trimoxazole and ciprofloxacin were much sensitive drugs in our study with statistical significance $(\mathrm{p}<0.05)$. Khatri et al. (2017) reported that higher MRSA sensitive to vancomycin followed by co-trimoxazole (84.2\%) [24].

In Nepal, due to unnecessary use of antibiotic without doctor's prescription, the emergence of antibiotic resistance has been increasing. People can purchase antibiotic directly from pharmaceuticals, which has led to antibiotic resistant strains of microorganisms. However, the emergence of antibiotic resistant bacteria continues to threaten

Page $11 / 17$ 
the ability to treat infections. Recently, antibiotic resistant pathogens have been emerging in community, which may increase the impact they have on populations.

Antimicrobial resistance is an innate feature of bacterial biofilms and biofilm formation is higher in MRSA [25]. The biofilm forming ability of isolated MRSA was performed by microtitre plate method, tube method and congo red agar method. The biofilm producing isolated MRSA by microtitre plate method was $51.2 \%$ and non-biofilm producer were $48.7 \%$. On the other hand $41.4 \%$ of isolated MRSA were shown biofilm producer by tube method. Congo red agar method reported $34.1 \%$ MRSA isolate as biofilm producer.

In our study the strong, moderate and weak biofilm producers by microtitre plate methods were $15.4 \%, 35.7 \%$ and $48.7 \%$ respectively. The strong, moderate and weak biofilm producer by tube method was $11.3 \%, 30 \%$ and $58.5 \%$ respectively. The strong, moderate and weak biofilm producers by congo red agar method were $8.9 \%, 25.2 \%$ and $65.8 \%$ respectively. In overall the biofilm producing CA-MRSA were $51.2 \%$ and rest (48.8\%) were non-producers. Number of false positive and false negative was reported in the comparison. It was difficult to discriminate strong, moderate and weak biofilm producers in tube method and congo red agar method due to phenotypic variations. The Sensitivity and specificity of tube method was found to be $75 \%$ and $74.6 \%$ respectively. For congo red agar method the sensitivity and specificity was found to be $54.8 \%$ and $85.2 \%$. The statistical analysis of screening were similar even in our findings which supports different other similar findings done before. Hassan et al. 2011 concluded microtitre plate method as gold standard technique for screening biofilm as compared to tube method and congo red agar method [12]. In our study, the microtitre plate method was found to be most sensitive and efficient method for quantitative screening of biofilm as compared to tube and congo red agar method. The screening of biofilm by microtitre method, tube method and congo red agar method was statistically significant $(p<0.05)$. The study conducted by Rezaei et al. (2013) reported that $15.4 \%$ of CA-MRSA was strong biofilm producer and rest $19.2 \%$ and $65.4 \%$ were medium and weak biofilm producer respectively [26].

The biofilm producing MRSA showed high resistance to cefotaxime, chloramphenicol, teicoplanin, co-trimoxazole, erythromycin, norfloxacin, trimethoprim. The non-biofilm producing MRSA showed resistance to tetracycline, ofloxacin, gentamicin, and ciprofloxacin. The resistivity pattern of biofilm producer and non-biofilm producer was statistically significant $(\mathrm{p}<0.05)$. Ampicillin and amoxicillin were resisted by both biofilm producers and non-biofilm producers. The resistant pattern of biofilm producing reported the ability of biofilm formation in drug resistance. However, non-biofilm producing antibiotic resistant was even found in our study. It can be explained that other than biofilm formation many other contributory role of bacteria is responsible for drug resistance.

Ciprofloxacin is known to be most effective against biofilm forming bacteria. In our study ciprofloxacin showed effectiveness to both biofilm producing and non-producing MRSA strains. In our study, only $1.5 \%$ biofilm producers were resistant to ciprofloxacin and rests were all sensitive. In our study the sensitivity of MRSA to ciprofloxacin was statistically significant $(p<0.05)$. The study conducted by ElShekh et al. (2013) reported that $99.9 \%$ of MRSA isolates were susceptible to ciprofloxacin and vancomycin [27]. Similar finding was seen even in our study. Most of the biofilm producing MRSA isolates showed higher resistance to trimethoprim and erythromycin. In overall $76.4 \%$ CA-MRSA were resistant to trimethoprim. Pate et al. 2009 reported that trimethoprim/sulfamethoxazole resistance associated with MRSA infections [28]. In our study the Trimethoprim resistance MRSA was $76.4 \%$ which shows increasing resistance pattern of CA-MRSA. The antimicrobial resistance seen in our study was higher among biofilm producing MRSA. The study conducted by Neopane et al. (2018) reported the higher drug resistance by biofilm producing Staphylococcus aureus than the non-producers $(p<0.05)$ [29]. The study indicates that biofilm producing ability might be one of the crucial factors for resistance towards the antibiotics.

Page $12 / 17$ 
In our study, $91 \%$ of CA-MRSA isolates were known to be MDR. Multidrug resistant bacteria were higher among biofilm producers which was also statistically significant $(p<0.05)$. The emergence of multi-drug resistant MRSA has further increased the need for search of alternative antimicrobial therapeutic agents in order to tackle with the infections caused by MDR CA-MRSA. The study strongly suggests adding up proper policy on sanitation,

precaution and awareness to prevent the infections of CA-MRSA. Multidrug resistance pattern may limit option for clinical therapy.

Our study reported a significant prevalence of VISA in nasal swab as well as in hand swab from all potential and healthy population. However, the prevalence of VISA in hand and nasal was not statistically significant $(p>0.05)$. In overall study population the $49.5 \%$ of MRSA were identified as VISA strains $(4-8 \mu \mathrm{g} / \mathrm{ml})$. Similarly, the study performed by Mendez et al. (2013) reported the nasal and hand surface colonized by VISA strains but showed lower prevalence of VISA (20\%) [30].

VRSA and VISA were detected in all potential risk population as well as in healthy controls. In the study, $40.65 \%$ of MRSA were identified as VRSA and the overall prevalence of VRSA in population was $25 \%$. The prevalence of VRSA in nasal cavity of study population was $11.5 \%$. The prevalence of VRSA was high in potential risk populations. In our Study $38 \%$ of MRSA isolates from barber population were identified as VRSA. The similar prevalence of VRSA strains were identified in beauticians (32\%). In our study the prevalence of VRSA and VISA strains in nasal cavity were $11.5 \%$. However, the study conducted by ElSayed et al. (2018) reported only one strain of VISA from nasal cavity of health care worker [31]. Ghoniem et al. 2014 reported higher resistance rate $20.68 \%$ VISA and $20.68 \%$ VRSA from clinical samples which indicates the rising prevalence of VRSA and VISA strains [32]. Although vancomycin has been used as a drug of choice for treating MRSA, recently the VISA has been reported in CA-MRSA clones [33].

Detection of vancomycin resistance is essential not only for an optimal therapy, but also for infection control measures and epidemiological purposes. As recommended by the Clinical \& Laboratory Standards Institute (CLSI), the broth dilution method was used for MIC determination for the detection of VISA and VRSA strains in this study. Most of the microbiological laboratories in Nepal depend upon disk diffusion method to identify susceptibility of $S$. aureus to vancomycin, which however cannot differentiate VISA and VRSA strains because of similar zone of inhibition. Detection of VRSA raises the concern and issue not only in clinical therapy but even in epidemiological settings.

\section{Conclusions}

In our study significant rise of VISA and VRSA were reported from CA-MRSA. The pathogen is spreading in community, hospitals and in wide areas. It can be warned that lack of hygiene, exposure to wastes and unauthorized use of drugs lead in transmission of the MRSA. These results concludes that emergence of VISA and VRSA in community setting requires immediate collaborative effort to search for measures to fight with the superbug. Improvement in personal hygiene and formulation of appropriate antimicrobial policy will help to prevent CA-MRSA infection.

\section{Declarations}

Principal investigator: Mr. Jenish Shakya, jenish11.js@gmail.com

Corresponding Author: Mr. Hemanta Khanal, khanal.hemanta@gmail.com

Page $13 / 17$ 
Mr. Prince Subba, princesubba05@gmail.com

Mr. Hemanta Khanal, khanal.hemanta@gmail.com

Source of Support: Nepal Academy of Science and Technology, Khumaltar, Lalitpur

Acknowledgements: The authors want to thank all the research participants and Microbiology Department of Central Campus of Technology, Tribhuvan University and Nepal Academy of Science and Technology, Khumaltar, Lalitpur for the financial support.

Conflict of interest: Authors declare no conflict of interest.

Ethics approval and consent of participation: The Research study was carried out after receiving ethical approval from Nepal Health Research Council, Kathmandu. Before starting this study the written Informed Consents were obtained from the participants.

\section{References}

1. Monecke S, Coombs G, Shore AC, Coleman DC, Akpaka P, Borg M, Chow H, Margaret Ip, Jatzwauk L, Jonas D, Kadlec K, Kearns A, Laurent F, O'Brien FG, Pearson J, Ruppelt A, Schwarz S, Scicluna E, Slickers P, Hui-Leen Tan, Weber S, Ehricht R. "A field guide to pandemic, epidemic and sporadic clones of methicillin-resistant Staphylococcus aureus," PLoS ONE. 2011; 6(4), e17936.

2. Wayne PA. USA: Clinical and Laboratory Standards Institute (CLSI). M100-S21: Performance standards for antimicrobial susceptibility testing. 2012; 32. 21 st Informational Supplement.

3. Moran GJ, Krishnadasan A, Gorwitz RJ, Gregory EF, McDougal LK, Carey RB, and Talan DA. Methicillin-resistant aureus infections among patients in the emergency department. N Engl J Med. 2006;355:666-74.

4. Chambers HF and Deleo FR. Waves of resistance: Staphylococcus aureus? Emerge Infect Dis; 2009;7:178-82.

5. Smyth R and Kahlmeter G. "Mannitol salt agar-cefoxitin combination as a screening medium for methicillinresistant Staphylococcus aureus." Journal of clinical microbiology. 2005;43(8): 3797-3799.

6. Finks J, Wells E, Dyke TL, Husain N, Plizga L, Heddurshetti R. Vancomycin-resistant Staphylococcus aureus, Michigan, USA, 2007. Emerg Infect Dis; 2009;15(6):943-5. doi: 10.3201/eid1506.081312.

7. Amatya R, Devkota P, Gautam A. Reduced susceptibility to vancomycin in methicillin resistant Staphylococcus aureus: a time for action. Nepal Med Coll J. 2014;16(1):42-4.

8. Clinical and laboratory standard institute. Performance standards for antimicrobial disc susceptibility tests, twentieth supplement. 2012;32(3): M100- S21.

9. Christensen GD, Simpson WA, Younger JJ, Baddour LM, Barrett FF, Melton DM, Beachey EH. Adherence of coagulase-negative Staphylococci to plastic tissue culture plates: a quantitative model for the adherence of staphylococci to medical devices. J Clin Microbiol. 1985;22(6):996-1006.

10. Stepanović, S, Vukovic D, Hola V, Bonaventura GD, Djukić S, Ćirković I and Ruzicka F. "Quantification of biofilm in microtitre plates: overview of testing conditions and practical recommendations for assessment of biofilm production by staphylococci." Apmis. 2007;115(8): 891-899. 
11. Freeman DJ, Falkiner FR, Keane CT. New method for detecting slime production by coagulase negative Staphylococci. J ClinPathol. 1989;42(8):872-4.

12. Hassan A, Usman J, Kaleem F, Omai M, Khalid A, Iqbal M. Evaluation of different detection methods of biofilm formation in the clinical isolates. Brazilian journal of infectious Diseases. 2011;15(4); 305-311.

13. Ansari S, Nepal HP, Gautam R, Rayamajhi N, Shrestha S, Upadhyay G, Acharya A and Chapagain ML. "Threat of drug resistant Staphylococcus aureus to health in Nepal." BMC infectious diseases. 2014;14(1): 157.

14. Forbes BA, Sahm DF, Weissfeld AS. Staphylococcus, Micrococcus and similar organisms. Bailey Scott's Diagn Microbiol. 2007;12(Chapter 16):254-63.

15. Edmond MB, Wenzel RP and Pasculle AW. Vancomycin-resistant Staphylococcus aureus: perspectives on measures needed for control. Ann Intern Med. 1996;329-334.

16. Rai SK, Tuladhar NR and Shrestha HG. Methicillin-resistant Staphylococcus aureus in a tertiary medical care center, Nepal. Ind J Med Microbial. 1990;8:108-9.

17. Kumari N, Mahopatra TM and Singh YI. Prevalence of MRSA in a tertiary care hospital in Eastern Nepal. J Nepal Med Assoc. 2008;47:53-6.

18. Rijal K, Pahari N, Shrestha B, Nepal A, Paudel B, Mahato P and Skalko-Basnet N. "Prevalence of methicillin resistant Staphylococcus aureus in school children of Pokhara." Nepal med coll J. 2008;10(3): 192-195.

19. Tiwari HK, Das A, Sapkota D, Sivrajan K, Pahwa V. Methicillin Resistant Staphylococcus aureus: Prevalence and antibiogram in a tertiary care hospital in western Nepal. J Infect DevCtries. 2009;3:681-684.

20. Khanal $L$ and Jha B. Prevalence of methicillin resistant Staphylococcus aureus (MRSA) among skin infection cases at a hospital in Chitwan, Nepal. Nepal Med Coll J 2010; 12(4): 224-228.

21. Mukhiya RK, Shrestha A, Rai SK. Prevalence of MRSA in hospitals of Kathmandu Valley. Nepal Journal of Science and Technology. 2012; 13(2): 185-90.

22. Wylie JL and Norwicki DL. Molecular Epidemiology of Community- and Health Care-Associated MethicillinResistant Staphylococcus aureus in Manitoba, Canada Journal of Clinical Microbiology. 2005;43 (6) 28302836; DOI:1128/JCM.43.6.2830-2836.

23. Mandelia C, Shenoy S and Garg Y. "Antibiotic sensitivity pattern of community associated-methicillin resistant Staphylococcus aureus." Revista da Socieda de Brasileira de Medicina Tropical. 2012;45(3): 418-418.

24. Khatri S, Pant ND, Bhandari R, Shrestha KL, Shrestha CD, Adhikari N and Poudel A. "Nasal Carriage Rate of Methicillin Resistant Staphylococcus aureus among Health Care Workers at a Tertiary Care Hospital in Kathmandu, Nepal." Journal of Nepal Health Research Council. 2017;15(1): 26-30.

25. CharanKaur D and Khare AS. Biofilm formation and antibiotic susceptibility pattern in MRSA strains in a tertiary care rural hospital. Indian Journal of Basic and Applied Medical Research. 2013;1(3), P. 37-44.

26. Rezaei M, Moniri R, Mousavi SGA and Shiade MJ. "Prevalence of biofilm formation among methicillin resistance Staphylococcus aureus isolated from nasal carriers." Jundishapur Journal of Microbiology. 2013;6(6).

27. El-Shekh NA, AyoubAM, El-Hendawy HH, Abada EA and Khalifa SY. "In vitro activity of some antimicrobial agents against intact and disrupted biofilms of staphylococci in the indwelling vascular catheter patients." World Appl Sci J. 2010;10(1): 108-120.

28. Pate AJ, Mokkarala VS, Patel N, Vernaleo J and Chendrasekhar A. "Trimethoprim-sulfamethoxazole resistance is associated with increased mortality in patients with methicillin-resistant Staphylococcus aureus" Chest. 2009;136(4): 11s.

Page 15/17 
29. Neopane P, Nepal HP, Shrestha R, Uehara O and Abiko Y. "In vitro biofilm formation by Staphylococcus aureus isolated from wounds of hospital-admitted patients and their association with antimicrobial resistance." International journal of general medicine. 2018;11: 25.

30. Méndez IA, Holguín-Riaño DF, Pachón-Barinas DP, Africano FJ, González IM and Rojas NA. "Prevalence and antimicrobial susceptibility of Staphylococcus aureus methicilin resistant isolated from medical students." CES Medicina. 2013;27: 21-30.

31. ElSayed N, Ashour M and Amine AEK. "Vancomycin resistance among Staphylococcus aureus isolates in a rural setting, Egypt." Germs. 2018;8(3): 134.

32. Ghoniem EM, El Hendawy GR, Moteleb TMA, Hassan HA and Khalil HAER. "Characterization of vancomycinresistant Staphylococcus aureus in the National Liver Institute." Menoufia Medical Journal. 2014;27(4): 825.

33. Graber CJ, Wong MK, Carleton HA, Perdreau-Remington F, Haller BL and Chambers HF. "Intermediate vancomycin susceptibility in a community-associated MRSA clone." Emerging infectious diseases. 2007;13(3): 491.

\section{Figures}

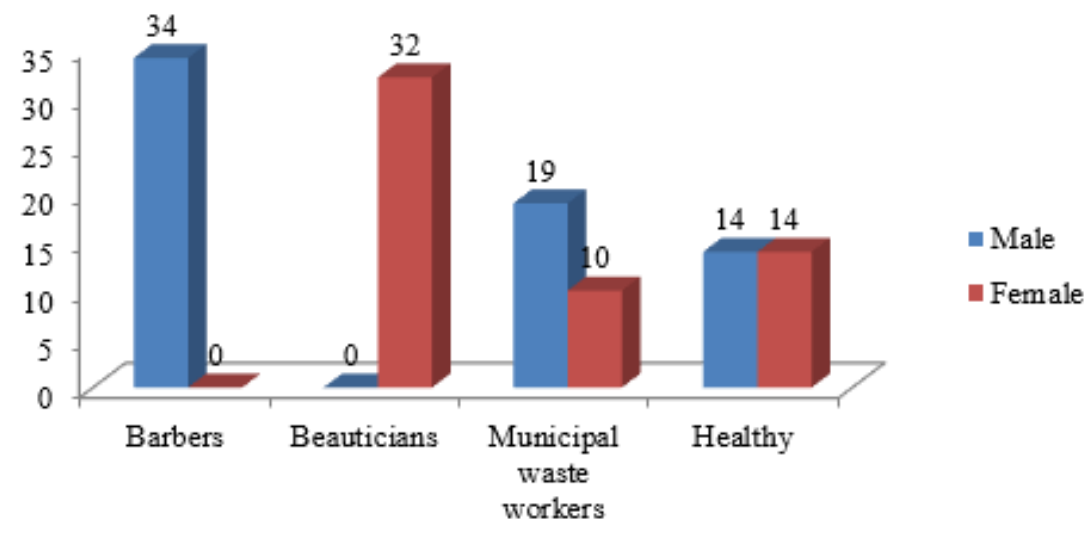

\section{Figure 1}

Gender wise distribution of MRSA positive in sample population

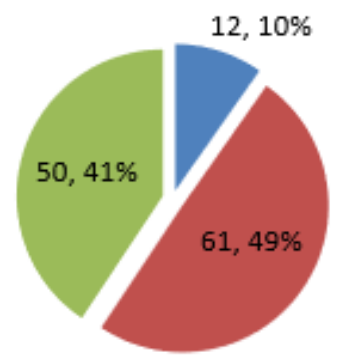

- VSSA $(\leq 2 \mu \mathrm{g} / \mathrm{ml})$

- VISA $(4-8 \mu \mathrm{g} / \mathrm{ml})$

VRSA $(\geq 16 \mu \mathrm{g} / \mathrm{ml}$

\section{Figure 2}

Vancomycin sensitivity of total MRSA isolates 


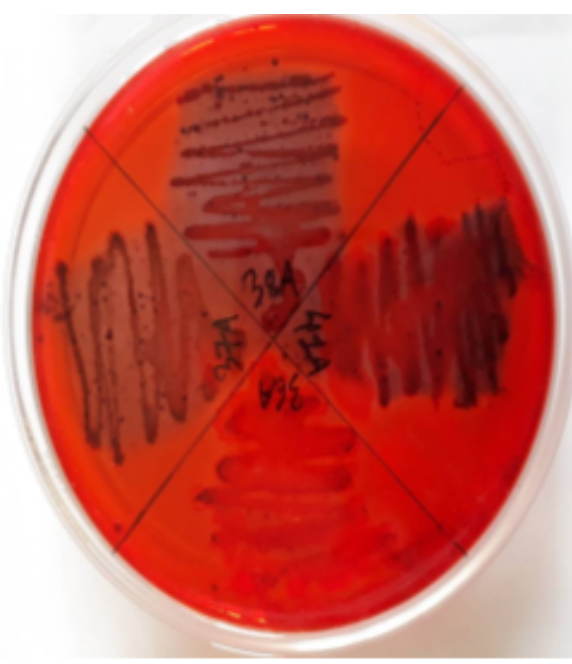

A

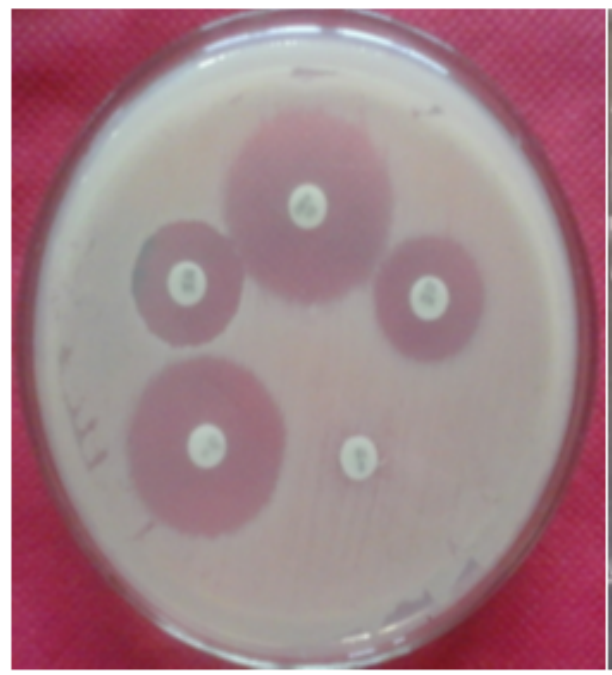

$\mathbf{C}$

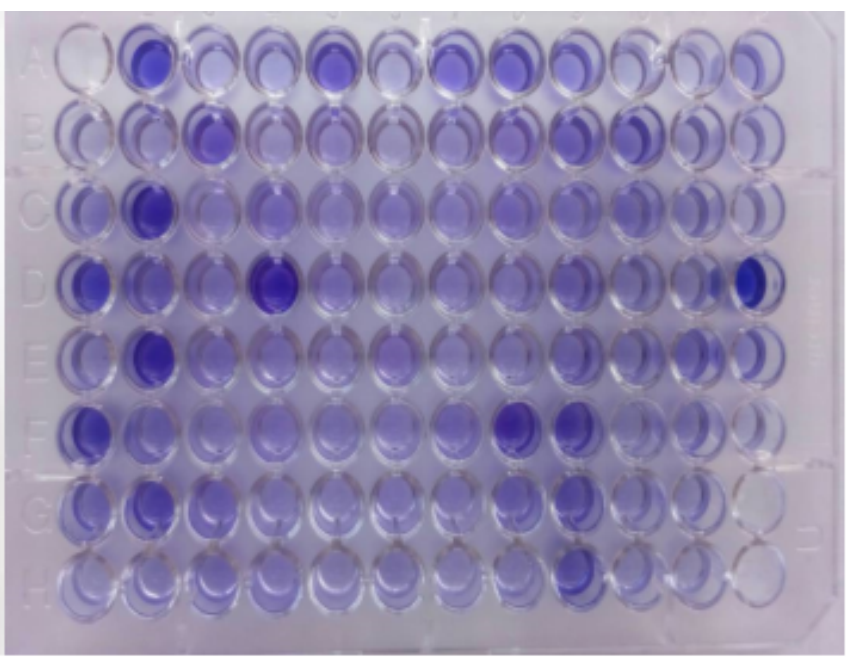

B

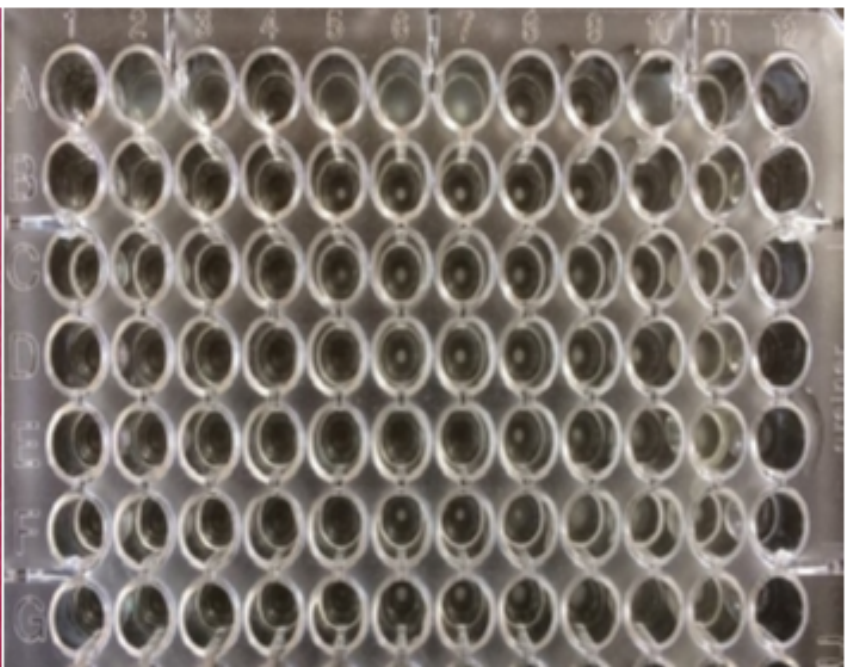

D

\section{Figure 3}

Different results of CA-MRSA. (A) Biofilm screening by congo red agar positive (black colonies). (B) Biofilm production of CA-MRSA in microtitre wells. (C) Antibiotic susceptibility test of CA-MRSA. (D) MIC of vancomycin by Microbroth dilution method. 